\title{
Design-Kriterien sprachsensiblen Geographieunterrichts
}

Kapitel 2 und 3 legen den Grundstein für die Design-Kriterien und die darauf basierende sprachsensible Unterrichtsreihe zum Thema Schalenbau der Erde und Plattentektonik. Dieses Thema ergibt sich aus dem Rahmenlehrplan der Berliner Schulen für die 7. Jahrgangsstufe. Es wird außerdem genuin in der Geographie unterrichtet und ist im Rahmenlehrplan für das Fach Geographie aller Länder verankert. Dieses Kapitel ist als Brücke zwischen dem Grundlagenteil auf der einen Seite und der eigenen empirischen Forschungsarbeit auf der anderen Seite zu verstehen. Die vier Design-Kriterien werden in den folgenden Unterkapiteln eingehender vorgestellt. Dabei wird aufgezeigt, wie sich die Kriterien ableiten und wie sie zu verstehen sind. Jedes Kapitel enthält einzelne Beispiele aus der sprachsensiblen Unterrichtsreihe, die veranschaulichen, wie das entsprechende Kriterium in der Praxis operationalisiert wurde. Die sprachsensible Unterrichtsreihe, auf die in diesem Kapitel Bezug genommen wird, ist als Intervention der Studie zentraler Forschungsgegenstand. Abb. 4.1 zeigt die Design-Kriterien sowie deren wesentliche Charakteristika stichpunktartig zusammengefasst.

Die Design-Kriterien sind als Handlungsmaxime für sprachsensiblen Geographieunterricht zu verstehen, die keinen Anspruch auf Vollständigkeit erheben und je nach Bedarf der Schüler*innen und Situation unterschiedlich priorisiert werden können und sollten. Während das erste Kriterium „Guter Geographieunterricht“ auch bei nicht-sprachsensibler Ausrichtung immer gegeben sein sollte, kann zum Beispiel der Einbezug der Erstsprache weniger explizit Einzug in den Unterricht finden. Bildlich gesprochen, ist guter Geographieunterricht das notwendige Fundament sprachsensiblen Geographieunterrichts, die weiteren Kriterien bilden die Säulen, die das Haus sprachsensiblen Geographieunterrichts trägt (vgl. Abb. 4.1).

Darüber hinaus werden in den obigen Ausführungen auch weitere Hinweise für sprachsensiblen Unterricht dargestellt. Insbesondere in vielen konzeptionellen 


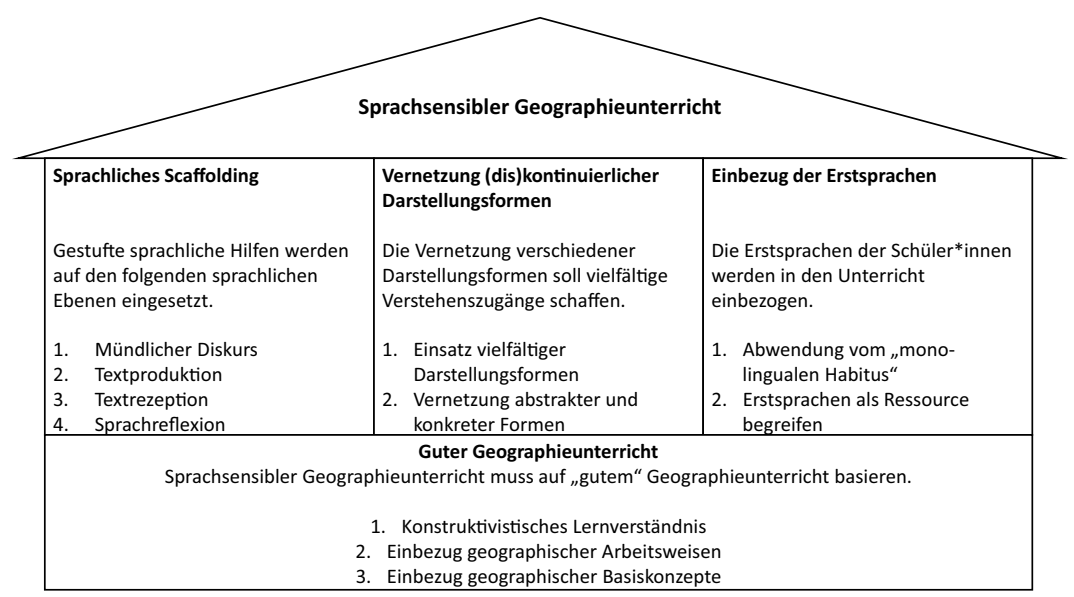

Abb.4.1 Design-Kriterien sprachsensiblen Geographieunterrichts in der Zusammenschau. (Eigene Darstellung)

Arbeiten sind Ideen für die Umsetzung gegeben, die in den Kriterien integriert sind. Doch auch die vorgestellten Design-Kriterien bieten eine Vielzahl an Operationalisierungsmöglichkeiten, die im Einzelnen stark variieren können.

\subsection{Guter Geographieunterricht als Basis}

Form follows function. Mit diesen drei Wörtern ist kurz zusammengefasst, warum guter Geographieunterricht das erste und gleichsam basale Design-Kriterium für sprachsensiblen Geographieunterricht ist. Jeder Geographieunterricht sollte das Ziel haben, Geographie möglichst gut $\mathrm{zu}$ unterrichten. Die Gestalt des Unterrichts, also die äußere Form, leitet sich dabei aus der Funktion ab (vgl. Abschn. 3.1). Wenn Geographieunterricht sprachsensibel gegeben wird, geht ihm die Annahme voraus, dass Geographie sprachsensibel besser gelernt werden kann als ohne sprachsensible Ausrichtung. Damit sprachsensibler Geographieunterricht (sowie sonstiger Geographieunterricht) gut sein kann, sollten möglichst die Kriterien guten Geographieunterrichts erfüllt sein. Was guter Geographieunterricht ist, ist allerdings weder normativ-konsensual gesetzt noch empirisch umfassend geklärt. Die Annäherung an guten Geographieunterricht in Abschnitt 3.1 ist maßgeblich für dieses Design-Kriterium. Im Folgenden werden die oben dargestellten 
Charakteristika guten Geographieunterrichts, die in Forschung und Unterrichtspraxis weitgehend auf Konsens stoßen, noch einmal knapp dargestellt sowie deren Operationalisierung mithilfe ausgewählter Unterrichtsmaterialien veranschaulicht. Dem vorangestellt sei der Hinweis, dass das Kriterium „Guter Geographieunterricht“ nicht nur für die sprachsensible Unterrichtsreihe von Belang ist. Auch die Vergleichsgruppe ohne sprachsensiblen Geographieunterricht erhielt Geographieunterricht, der auf den Kriterien guten Geographieunterrichts basiert (vgl. Kap. 6).

Zentral für guten Geographieunterrichts ist ein ihm zugrundeliegendes konstruktivistisches Lernverständnis (vgl. Abschn. 3.1). Dazu gehört inhaltlich der Einbezug domänenspezifischer Schüler*innenvorstellungen (vgl. Abb. 4.2). Methodisch wird möglichst viel kognitive Aktivierung auf Schüler*innenseite und konstruktivistische Unterstützung von Lehrer*innenseite gefordert. Das bedeutet, die Informationen werden von den Lernenden aktiv auf Basis des jeweiligen Vorwissens konstruiert, auch wenn die Lehrkraft Material als Informationsbereitstellung einbringt. Der Lernprozess und auch das Lernergebnis variieren also von Schüler*in zu Schüler*in. In diesem Sinne ist Lernen in hohem Maße individuell und hängt von den Systemen der Lernenden ab, insofern ist immer das jeweilige Vorwissen der Schüler*innen zu berücksichtigen. Der Lernprozess kann zwar von der Umgebung ausgelöst werden, das Lernen selbst läuft allerdings selbstdeterminiert ab. Hierfür werden individuelle Hilfen, Vorstrukturierungen etc. für die*den Lernende*n benötigt. Das folgende Beispiel zeigt die Aktivierung von Vorwissen und eigenen Vorstellungen und gibt gleichzeitig sprachliche Hilfen zur Formulierung (vgl. Abb. 4.2).

Lernen ist außerdem sozial eingebettet, da es immer in sozialer Interaktion stattfindet. Verschiedene Sozialformen sollten also im Rahmen des Unterrichts Berücksichtigung finden. Lernen ist situiert, also immer kontextgebunden. Die Kontexte und Beispiele, an und in denen gelernt wird, sollten variieren und flexibel eingesetzt werden. Sie sollten so gewählt werden, dass sie für die Schüler*innen alltagsnahe und realistische Situationen abbilden (vgl. Kestler 2014, S. 55-56; Otto 2012, S. 47-48; Reinfried 2015, S. 56). Der Anteil an Schüler*innenaktivitäten ist in beiden Unterrichtsreihen (Experimental- und Kontrollgruppe) annährend gleich hoch; ebenfalls variieren bei beiden die Sozialformen.

Neben der konstruktivistischen Ausrichtung des Unterrichts ist in der Geographie der Einbezug geographischer Arbeitsweisen gefordert. Dem wird in beiden Unterrichtsreihen mit Atlas-, Karten-, Diagrammarbeit sowie dem Einbezug von 


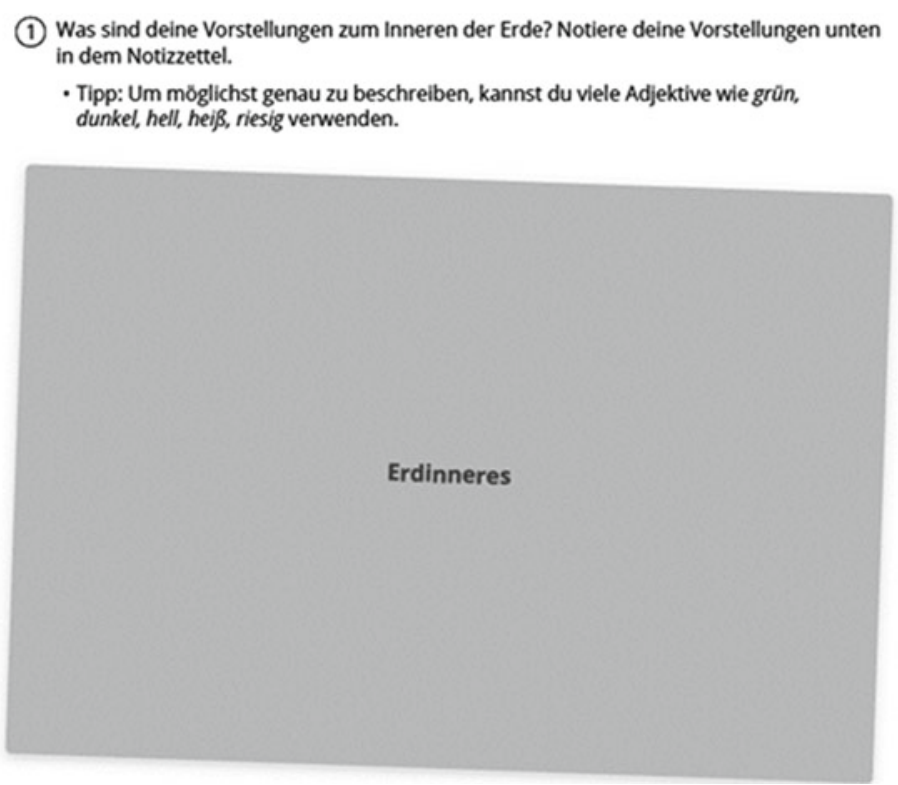

Abb.4.2 Beispiel für den Einbezug von Schüler*innenvorstellungen. (Eigene Darstellung)

Modellen beigekommen (vgl. Wilhelm et al. 2018, S. 16). Darüber hinaus enthalten beide Unterrichtsreihen in gleichem Maße verschiedene Dimensionen der geographischen Basiskonzepte.

\subsection{Sprachliches Scaffolding}

Das zentrale Design-Kriterium für sprachsensiblen Geographieunterricht ist sprachliches Scaffolding. Gestufte sprachliche Hilfen werden bereitgestellt, damit das nächsthöhere Sprachniveau schließlich auch ohne Hilfen erreicht wird. In den vorangegangenen Kapiteln wird immer wieder auf das Scaffolding als zentralen Schlüssel für durchgängige Sprachbildung Bezug genommen (vgl. Abschn. 2.3, 3.3). So zeigen diverse Studien erfolgsversprechende Ergebnisse beim Einsatz eines am Scaffolding orientierten Unterrichts in Hinsicht auf das sprachliche und fachliche Lernen (vgl. Abschn. 2.3). Auch in den Fachdidaktiken hat sich Scaffolding im Rahmen konstruktivistischen Lernverständnisses in 
verschiedenen empirischen Studien als sinnvoller Weg durchgängiger Sprachbildung herauskristallisiert (vgl. Abschn. 3.3). Außerdem handelt es sich um einen konstruktivistischen Lernansatz, der hohe Passung mit dem Anspruch an guten Geographieunterricht aufweist (vgl. Abschn. 3.1 und 4.1).

Scaffolding gliedert sich in Makro- und Mikro-Scaffolding. Ersteres betrifft die Unterrichtsplanung und -materialien; letzteres meint die Unterrichtsinteraktion und ist weniger gut planbar. Aus diesem Grund wird im Rahmen der Studie und auch in diesem Design-Kriterium der Fokus auf das Makro-Scaffolding gelegt. In der sprachsensiblen Unterrichtseinheit wird auf Stationenlernen zurückgegriffen, was eben diesen Fokus auf der Unterrichtsplanung widerspiegelt. Dies ermöglicht eine bessere Vergleichbarkeit unter den Klassen unabhängiger von den Vorerfahrungen der Lehrkräfte, die insbesondere bei der Führung von Unterrichtsgesprächen sehr unterschiedlich sein kann. Scaffolds werden in den oben dargestellten Feldern der Sprachdidaktik gegeben (mündlicher Diskurs, Textproduktion, Textrezeption, Sprachreflexion).

\section{Mündlicher Diskurs}

Das Mikro-Scaffolding betrifft in erster Linie den mündlichen Unterrichtsdiskurs. Unter 3.3 wurde bereits eine Modellierungsmöglichkeit von Unterrichtsgesprächen nach Geist/Krafft (2017) vorgeschlagen, die in die sprachsensible Unterrichtsreihe integriert werden soll. Besonderes Augenmerk sei auf korrektives Feedback gelegt. Mit der Forderung nach korrektivem Feedback geht die Output-Orientierung einher, die kognitionspsychologisch, in der Spracherwerbsforschung und der Sprachdidaktik gefordert wird. Auch wenn dem Mikro-Scaffolding großes Potenzial zugeschrieben wird, fokussiert das Treatment dieser Studie auf das Makro-Scaffolding. Das heißt, der mündliche Unterrichtsdiskurs wird im Rahmen der Studie nicht erhoben. In der Schulung zur sprachsensiblen Unterrichtsreihe (Experimentalgruppe) wird den Lehrkräften das Mikro-Scaffolding erläutert und es wird auf die Bedeutsamkeit des Mikro-Scaffoldings hingewiesen, jedoch bräuchte es ein ausführliches Training, um - unabhängig von Vorerfahrungen mit Unterrichtsgesprächen - MikroScaffolding in dem Rahmen einzusetzen, wie es nötig wäre. Dieser Aspekt schwankt von Lehrkraft zu Lehrkraft zu stark, als er den Gütekriterien entsprechend hätte erhoben werden können.

\section{Textproduktion}

Schwerpunktmäßig fokussiert die Unterrichtsreihe Textproduktion. Dafür gibt es für die Schüler*innen gestufte Hilfen im Sinne des Scaffoldings. Beispielhaft sind die 
Sprachhilfen für folgende Aufgabe abgebildet: „Im neuen Geografiebuch ${ }^{1}$ fehlt ein passender Text zu Abb. 1.1 auf Seite 1. Schreibe einen Schulbuchtext zum Thema ,Schalenbau der Erde', in dem du die Abbildung beschreibst." (vgl. Abb. 4.3). Diese Aufgabe erhält neben dem Scaffolding auch Darstellungsvernetzung, indem Bild und Textproduktion miteinander verwoben werden. Darüber hinaus enthält die Unterrichtsreihe zahlreiche weitere gestufte Hilfen zur Textproduktion.

Die Einleitung schreiben

\begin{tabular}{|c|c|c|c|c|}
\hline \multirow{6}{*}{ 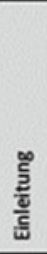 } & \multirow{3}{*}{ Abbildung 1 mit dem Titel „..." } & zeigt & $\begin{array}{l}\text { die verschledenen Schichten/Schalen } \\
\text { der Erde. }\end{array}$ & \\
\hline & & stellt & $\begin{array}{l}\text { die verschiedenen Schichten/Schalen } \\
\text { der Erde }\end{array}$ & dar. \\
\hline & & bildet & $\begin{array}{l}\text { die verschledenen Schichten/Schalen } \\
\text { der Erde }\end{array}$ & ab. \\
\hline & \multirow{3}{*}{ Auf Abbildung 1 mit dem Titel } & \multirow{3}{*}{ werden } & \multirow{3}{*}{$\begin{array}{l}\text { die verschiedenen Schichten/Schalen } \\
\text { der Erde }\end{array}$} & dargestellt. \\
\hline & & & & gezeigt. \\
\hline & & & & abgebildet. \\
\hline
\end{tabular}

\section{Den Hauptteil schreiben}

\begin{tabular}{|c|c|c|c|c|}
\hline \multirow{18}{*}{ 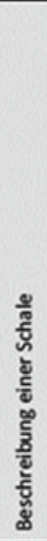 } & \multirow{4}{*}{$\begin{array}{l}\text { Die außerste / zweite / innerste } \\
\text { [...] Schicht/Schale }\end{array}$} & ist & der oberer Erdmantel [...]. & \\
\hline & & nennt sich & Erdkruste [...]. & \\
\hline & & heiBt & innerer Erdkern [....]. & \\
\hline & & wird & Erdkruste [...] & genannt. \\
\hline & \multirow{2}{*}{$\begin{array}{l}\text { Die Schale/Die Schicht } \\
\text { Die Erdkruste/Der Erdmantel } \\
\text { Sie/Er }\end{array}$} & ist & fest / teils fest, teils flussig [...]. & \\
\hline & & besteht aus & festem / zăhflussigem [...] Gestein. & \\
\hline & \multirow{4}{*}{$\begin{array}{l}\text { Die Schale/Die Schicht } \\
\text { Die Erdkruste/Der Erdmantel } \\
\text { Sie/Er }\end{array}$} & umfasst & eine Dicke von ... km. & \\
\hline & & ist & ... km dick. & \\
\hline & & befindet sich & In einer Tiefe zwischen ... und ... km. & \\
\hline & & reicht & bis in eine Tiefe von ... km. & \\
\hline & \multirow{4}{*}{$\begin{array}{l}\text { Dort } \\
\text { In dieser Schale/Schicht }\end{array}$} & betrăgt & die Temperatur bis zu / maximal ... " $\mathrm{C}$. & \\
\hline & & herrschen & Temperaturen von bis zu _. ${ }^{\circ} \mathrm{C}$. & \\
\hline & & kann & die Temperatur bis auf ... ${ }^{\circ} \mathrm{C}$ & ansteigen. \\
\hline & & kann & die Temperatur bis zu / maximal ... ${ }^{\circ} \mathrm{C}$ & betragen. \\
\hline & \multirow{4}{*}{$\begin{array}{l}\text { Die Temperatur in dieser } \\
\text { Schicht/Schale }\end{array}$} & betrăgt & maximal ... ${ }^{\circ} \mathrm{C}$ & \\
\hline & & kann & maximal _.. ${ }^{\circ} \mathrm{C}$ & betragen. \\
\hline & & steigt & \multirow{2}{*}{ auf bis zu ... ${ }^{\circ} \mathrm{C}$} & an. \\
\hline & & kann & & ansteigen. \\
\hline
\end{tabular}

Abb.4.3 Beispiel für Scaffolding auf Ebene der Textproduktion. (Eigene Darstellung)

\footnotetext{
${ }^{1}$ In der vorliegenden Arbeit wird die Schreibweise von Geographie mit $p h$ bevorzugt. Da das Land Berlin allerdings Geographie mit $f$ schreibt, wurde für die Unterrichtsmaterialien auf die Schreibung mit $f$ zurückgegriffen.
} 


\section{Textrezeption}

Neben der Textproduktion spielt auch die Textrezeption in der Unterrichtsreihe und ganz allgemein im Geographieunterricht eine entscheidende Rolle. In den Texten sind Hilfen enthalten, die durch Vorstrukturierung und Verständnisfragen auf Wortund Textebene Entlastung bieten sollen (vgl. Abb. 4.4).

\section{Platten bewegen sich voneinander weg: Divergenz}

(2) Lies den folgenden Text zunächst alleine. Kläre dann mit deinem Nachbarn oder deiner Nachbarin alle Wörter und Sätze, die du nicht verstehst.

Durch die Konvektionsströme wird die Erdkruste in verschiedene Richtungen bewegt. Man spricht von divergierenden Platten. Dort, wo die Platten auseinander gehen, kann Lava aufsteigen. Wenn die Lava erkaltet, entsteht ein neues Stück Erdkruste. Es können unterschiedliche Formen entstehen:

$5 \quad 1$. Wenn sich zwei kontinentale Platten voneinander weg bewegen, entsteht ein Grabenbruch.

2. Wenn sich zwei ozeanische Platten auseinander bewegen, entstehen mittelozeanische Rücken. Den Prozess nennt man seofloor-spreading.

Abb.4.4 Beispiel für Scaffolding auf Ebene der Textrezeption. (Eigene Darstellung)

\section{Sprachreflexion}

Unter Berücksichtigung des Vorwissens aller Schüler*innen geht dem Unterricht die Spezifikation und Explikation von sprachlichen Lernzielen voraus. Fachsprache kann nicht als gegebene Kompetenz bei allen vorausgesetzt werden, sondern muss als Lernziel formuliert werden, damit sie als Lerngegenstand wahrgenommen werden kann. Es ist erforderlich, dass Lehrkräfte schon zu Beginn einer Unterrichtsstunde oder -reihe sprachliche Ziele benennen, damit die Schüler*innen über ihre Fortschritte oder möglichen Lücken im Bilde sind (vgl. Schmölzer-Eibinger et al. 2013, S. 41). Im Rahmen der untersuchten Unterrichtsreihe werden auch die sprachlichen Lernziele zu Beginn der Unterrichtsreihe explizit gemacht (vgl. Abb. 4.5). Denn nur so ist es letztlich auch möglich, dass Reflexion über den sprachlichen Lernprozess stattfinden kann.

Ziel sprachsensiblen Unterrichts ist es, Sprachaufmerksamkeit zu erlangen. Diese „kann im Fachunterricht durch ein präzises und bewusst gestaltetes sprachliches Handeln der Lehrkräfte angeregt werden, aber auch durch ein Bewusstmachen und Reflektieren von sprachlichen Phänomenen, Begriffen oder Strukturen“ 


\section{Sprachliche Ziele der nächsten Geo-Stunden}

1. Du kannst besser verstehen, was deine Geografielehrerin/dein Geografielehrer sagt

2. Du kannst die Aufgaben im Geografieunterricht gut verstehen.

3. Du kannst dich mit deinen Mitschüler*innen über Geo-Themen unterhalten.

4. Du kannst Texte im Geounterricht besser verstehen.

5. Du übst, Texte im Geounterricht zu schreiben.

6. Du kannst mehr Wörter im Geografieunterricht verstehen.

7. Du kannst Tabellen und Diagramme im Geografieunterricht besser verstehen.

8. Du bekommst sorachliche Hilfe. wenn du sie brauchst.

Abb.4.5 Beispiel für Scaffolding auf Ebene der Sprachreflexion. (Eigene Darstellung)

(Schmölzer-Eibinger et al. 2013, S. 26). Dienlich für eine gelungene Sprachreflexion ist zum Beispiel das genaue Betrachten von sprachlichen Wendungen oder die rezeptive Auseinandersetzung mit Fachtexten.

Auch ist es sinnvoll, wenn Schüler*innen die Gelegenheit bekommen, über Leerstellen im Text zu reflektieren. Gedankenanstöße aufseiten der Lehrkraft können hierfür sehr hilfreich sein. Es ist oft wertvoll, Texte eingehender zu rezipieren, über feine Bedeutungsunterschiede zweier Fachbegriffe zu reflektieren und Details zu beachten. Die im Konstruktivismus geforderte selbstständige Bearbeitung von fachsprachlichen Aufgaben erfordert zusätzliche Hilfen wie Wörterbücher, Wortschatzkarten, gezielte Aufgaben oder das Internet, durch die die Schüler*innen unterstützt werden (vgl. Abschn. 3.3). Die Anwesenheit solcher Medien im Klassenzimmer ist nicht nur hilfreich, sondern markiert auch sichtbar für alle Lehrenden und Lernenden, dass Sprache und deren Förderung Teil eines jeden Faches ist.

\subsection{Vernetzung (dis)kontinuierlicher Darstellungsformen}

There is likely to be considerable message redundancy - that is, similar ideas will be expressed in a variety of different ways. Asking questions, exchanging information, and solving problems all provide a context where words are repeated, ideas are rephrased, problems are restated, and meanings are refined. This redundancy supports comprehension, because it gives learners several opportunities to hear a similar idea expressed in a number of ways. (Gibbons/Cummins 2002, 17 f.)

Die Darstellungsvernetzung beruht auf konstruktivistischem Lernverständnis und ist eine der zentralen Konstituenten sprachsensiblen Geographieunterrichts (vgl. 
Abschn. 3.1). Sie wird in der Sprachdidaktik, insbesondere bei der Textrezeption, als ein Schlüssel zum Verständnis betrachtet (vgl. Abschn. 3.3). Es sollen verschiedene Darstellungsformen für das Erklären desselben Inhalts verwendet werden, um den Schüler*innen eine große Bandbreite an Verstehenszugängen zu ermöglichen und diese dann auch miteinander zu verknüpfen. Erkenntnisse aus der Mathematikdidaktik deuten auf positive Effekte von Darstellungsvernetzung auf Verstehensprozesse hin (vgl. Abschn. 2.3). Die unterschiedlichen Darstellungsformen erschließen sich jedem*r Lernenden auf individuelle Weise. Jede hat dabei zugleich ihre fachlich begründeten spezifischen Vor- und Nachteile. Es liegt deshalb insbesondere im Fach Geographie aufgrund der hohen Anzahl an diskontinuierlichen Darstellungsformen nahe, diese Vielfalt als Ressource und nicht als Hürde zu begreifen, denn Darstellungsformen sind immer Mittel zur Verbalisierung fachlicher Sachverhalte (vgl. Abschn. 2.2). Sie arbeiten „,einander wechselseitig zu; häufig erweist sich sogar deren Wechsel als didaktischer Schlüssel zum fachlichen Verstehen" (Leisen 2013, S. 34). Ferner hilft die Verwendung unterschiedlicher Darstellungsformen dabei, die von den Bildungsstandards geforderte Kompetenz, in der Schule erworbenes Wissen auf die Alltagswelt zu übertragen, zu erfüllen. Die Darstellung in Bild, Sprache, Symbol oder mathematischer Formel kann es einfacher machen, Inhalte von fachlichen Kontexten auf alltägliche zu übertragen und umgekehrt (vgl. Leisen 2013, 34 f.). Es gewöhnt die Lernenden an den Umgang mit Fachwissen im Alltag, ermöglicht ihnen den fachlichen Verstehensprozess, auch unabhängig sprachlicher Verbalisierung und bietet so gleichzeitig auch die Chance fachsprachliche Strukturen anhand der Darstellungsformen explizit zu üben. Es sollten sowohl abstrakte als auch konkrete Darstellungsformen verwendet werden; nur Bilder anstatt von Texten oder Symbolen zu verwenden, führt weder $\mathrm{zu}$ besserem Verständnis noch zu höherer, weil umfassenderen, Kompetenz (vgl. Beese et al. 2014, S. 123). Abb. 4.6 zeigt die Vernetzung von Modell, Text und Abbildung. In der Unterrichtsreihe kommen diverse weitere vor, so insbesondere auch die Vernetzung von Karte und Textproduktion oder -rezeption.

\subsection{Einbezug der Erstsprache}

Die Abwendung vom monolingualen Habitus etabliert sich zunehmend, wenn auch zögerlich, in deutschen Schulen. Sprachsensibler Geographieunterricht bezieht die Erstsprache, wenn möglich und sinnvoll, aktiv in den Unterricht mit ein. In einer Interviewstudie von Riegger et al. (2017) mit 94 Schüler*innen zeigt sich, dass starke emotionale Bezüge zur Erstsprache sowie Bedürfnisse 
Der oberste Teil der Erde ist in eine feste und eine zăhflüssige Schicht eingeteilt. Die zähflüssige Schicht bewegt sich.

(1) Schaue das Video zu „Konvektionsströmen“ an.

(2) Wie bewegt sich die Tinte im Modell?

a) Zeichne mit Pfeilen in Abb. 1 ein, wie sich die Tinte im Video bewegt.

b) Beschreibe, wie sich die Tinte im Video bewegt.

c) Korrigiere dann mit dem Löungsblatt.
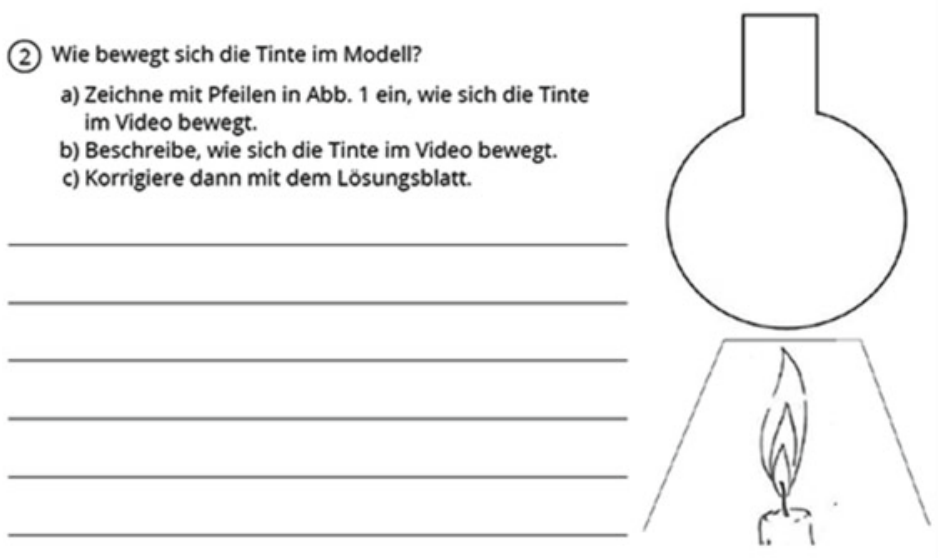

Abb. 1 - Versuchsaufbau

Abb.4.6 Beispiel für Darstellungsvernetzung. (Eigene Darstellung)

zum aktiven Einbezug der Sprache im Alltag bestehen (Riegger et al. 2017, S. 161). Dies sowie verschiedene Spracherwerbstheorien legen nahe, dass der Einbezug von Mehrsprachigkeit als Ressource genutzt werden kann und sollte (vgl. Abschn. 3.2). So legt die am Behaviorismus orientierte Kontrastivhypothese nahe, dass mögliche sprachliche Verständnis- und Fehlerquellen in der Zweitsprache in den Strukturen der Erstsprache liegen. Der Einbezug der Erstsprache ist also besonders dann lohnend, wenn die*der Lernende in der Erstsprache auch fach- und bildungssprachlich kompetent ist. Außerdem bietet ein Grundverständnis der Lehrperson über die Strukturen der Erstsprachen der Schüler*innen die Möglichkeit, Fehlerquellen zu entdecken und entsprechend entgegensteuern zu können. Die fehlerbehaftete Übertragung von Gewohnheiten der Erstsprache auf die Zweitsprache können so vermieden werden. Auch die Schwellenhypothese macht den Einbezug der Zweitsprache evident, denn demgemäß muss für den Erwerb bildungs- und fachsprachlicher Kompetenzen in der Zweitsprache auch die alltagssprachliche Kompetenz in der Zweitsprache ausreichend ausgebildet sein. In diesem Zusammenhang kann der Einbezug der Erstsprache zum Erwerb fach- und bildungssprachlicher Kompetenzen in der Zweitsprache lohnend sein, 
v. a. sofern die fach- und bildungssprachliche Kompetenz in der Erstsprache bereits gut ausgebildet ist (vgl. Abschn. 3.2). Im Rahmen der untersuchten Unterrichtsreihe werden die Schüler*innen dazu eingeladen, ihre Erstsprache oder weitere Sprachen als Ressourcen zu begreifen, die ihnen beim fachlichen Verständnis helfen können, indem explizit Raum für die Erstsprache gegeben wird (vgl. Abb. 4.7).

\section{Hinweise zur Stationenarbeit}

1. Bringe zu jeder Stunde dein Material mit.

2. Arbeite ruhig, damit du niemanden störst.

3. Bearbeite die Stationen 1-5 in der richtigen Reihenfolge.

4. Wie viel Zeit du für jede Station einplanen sollst, siehst du in der Tabelle.

5. Die Lösungen für die Aufgaben bekommst du vorne am Pult.

6. Du darfst ein Wörterbuch verwenden.

7. Du kannst dich über die Geografie-Inhalte auch in einer anderen Sprache als Deutsch unterhalten, wenn du die Aufgaben dann besser verstehst.

8. Manche Aufgaben sind freiwillig, manche Aufgaben Pflicht. Aufgaben mit dem Wort EXTRA machst du nur, wenn du genug Zeit hast.

Abb.4.7 Beispiel für den Einbezug der Erstsprachen. (Eigene Darstellung)

Wie explizit die Erstsprache einbezogen wird, hängt von verschiedenen Faktoren ab. Nicht zuletzt von der sprachlichen Kompetenz der Lehrperson. Eben weil es völlig illusorisch ist, dass diese die Erstsprachen ihrer Schüler*innen beherrscht, wird die Erstsprache in bisherigen Ansätzen sprachsensiblen Fachunterrichts selten empfohlen. Diese Arbeit macht den Einbezug der Erstsprache zu einem von vier Design-Kriterien.

Aus den Kapiteln 2 und 3 wurden vier Design-Kriterien für sprachsensiblen Geographieunterricht abgeleitet. Für die Design-Kriterien wurden Erkenntnisse aus der Geographie- und Sprachdidaktik sowie aus anderen Fachdidaktiken, in denen die Forschung bereits weiter gediehen ist (insbesondere der Mathematik und den Naturwissenschaften) einbezogen. Die Kriterien können auf unterschiedliche Weise operationalisiert und priorisiert werden und erheben keinen Anspruch auf Vollständigkeit. Die Kriterien 
1) Guter Geographieunterricht als Basis, 2) Sprachliches Scaffolding, 3) Vernetzung (dis)kontinuierlicher Darstellungsformen und 4) Einbezug der Erstsprache bilden die Basis für die beforschte Unterrichtsreihe.

Open Access Dieses Kapitel wird unter der Creative Commons Namensnennung 4.0 International Lizenz (http://creativecommons.org/licenses/by/4.0/deed.de) veröffentlicht, welche die Nutzung, Vervielfältigung, Bearbeitung, Verbreitung und Wiedergabe in jeglichem Medium und Format erlaubt, sofern Sie den/die ursprünglichen Autor(en) und die Quelle ordnungsgemäß nennen, einen Link zur Creative Commons Lizenz beifügen und angeben, ob Änderungen vorgenommen wurden.

Die in diesem Kapitel enthaltenen Bilder und sonstiges Drittmaterial unterliegen ebenfalls der genannten Creative Commons Lizenz, sofern sich aus der Abbildungslegende nichts anderes ergibt. Sofern das betreffende Material nicht unter der genannten Creative Commons Lizenz steht und die betreffende Handlung nicht nach gesetzlichen Vorschriften erlaubt ist, ist für die oben aufgeführten Weiterverwendungen des Materials die Einwilligung des jeweiligen Rechteinhabers einzuholen.

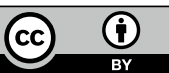

